\title{
Imaging of meniscal cyst of the knee in three cases
}

\author{
Anthony Coral, M.B.B.S., M.R.C.P., F.R.C.R. ${ }^{1}$, M. van Holsbeeck, M.D. ${ }^{2}$, \\ R.S. Adler, Ph.D., M.D. ${ }^{1}$ \\ ${ }^{1}$ Department of Radiology, University of Michigan Health Center, Ann Arbor, Michigan, USA \\ ${ }^{2}$ Department of Radiology, University Hospitals K.U., Leuven, Belgium.
}

\begin{abstract}
Three cases of solitary meniscal cyst in the knee have recently been diagnosed in our departments using ultrasound and nuclear magnetic resonance (MRI). Two cysts involved the lateral and one the medial meniscus. The appearance of these lesions on ultrasound and MR images is shown. All three cysts had low intensity on T1-weighted images and high intensity on T2-weighted images and clearly communicated with a large horizontal tear in the meniscus. Ultrasonography showed a relatively hypoechoic lesion and, in the two cases of lateral meniscal cyst, focal areas of relatively increased echoes which are probably due to meniscal debris. Ultrasound showed the abnormal meniscus in both of these cases. MRI is the best way of showing the cyst and an accompanying meniscal tear but, where MRI is not available, ultrasound may be an easy and inexpensive way of making a diagnosis whether or not it is used in conjunction with positive contrast arthrography.
\end{abstract}

Key words: Meniscal cyst - Ultrasound - Magnetic resonance

Ultrasonography, computed tomography and magnetic resonance imaging (MRI) have made it easier to diagnose the cause of a swelling around the knee. Meniscal cysts are lesions of the semilunar fibrocartilage of the knee that were formerly believed to be uncommon. They were present in an estimated $1 \%$ of all meniscectomies performed at the Mayo Clinic (50 patients with 51 cysts) over a 28 year period (up to 1962) [2] and in $2 \%$ of a series of 2522 arthrograms [14]. The incidence of cystic change in surgically removed menisci is, however, much higher than these figures if all areas of cystic change, including those

Address reprint requests to: Anthony Coral, M. B., Department of Radiology, University of Michigan Health Center, 1500 East Medical Center Drive, Ann Arbor, MI 48109-0326, USA under $0.3 \mathrm{~mm}$, are considered. Barrie found that 112 (7.1\%) of 1571 menisci showed cystic changes [1]. The largest series of meniscal pathological specimens are those of Smillie, who found that $145(2.5 \%)$ of 5,702 medial menisci and $488(22.2 \%)$ of 2,298 lateral menisci had cystic degeneration, with an overall incidence for both of $7.9 \%$, a figure similar to Barrie's [17]. The conclusion is that large, clinically recognizable lesions are relatively uncommon, whereas cystic changes are commonly observed on close inspection and microscopy of a surgically removed torn cartilage [3]. Of the 50 cysts found in 2522 arthrograms, 38 $(76 \%)$ had a clinically palpable lesion; however, arthrography is likely to detect only the larger cysts.

The usual presenting symptom of a meniscal cyst is a mass to the side of the femorotibial joint [2], but as nearly all patients admit to associated pain these cysts may also be found on MRI being done for suspected meniscal tears [5]. Cysts are well shown by MRI and have a characteristic appearance [5], but there are no previous reports of the use of ultrasound in their diagnosis. In two major series, meniscal cysts have been associated with a horizontally oriented tear in the meniscus in every case $[1,14]$. The cysts give an intense signal on $\mathrm{T} 2$-weighted scans and are often divided by septa. The tears are often best seen on T1-weighted images [5]. This is a report on the findings of ultrasound and MRI in three cases of cyst of the meniscus, two of which involved the lateral and one the medial meniscus.

\section{Case reports}

\section{Case 1}

A 30-year-old male soccer player had a painful swelling over the medial aspect of the right knee which had been present for 5 months. A clinical diagnosis of medial collateral ligament tear had been made, but sonography of the ligament showed a hypoechoic lesion adjacent to the meniscus (Fig. 1). Arthrography showed contrast medium in a serpentine, horizontal tear in the medial meniscus which communi- 

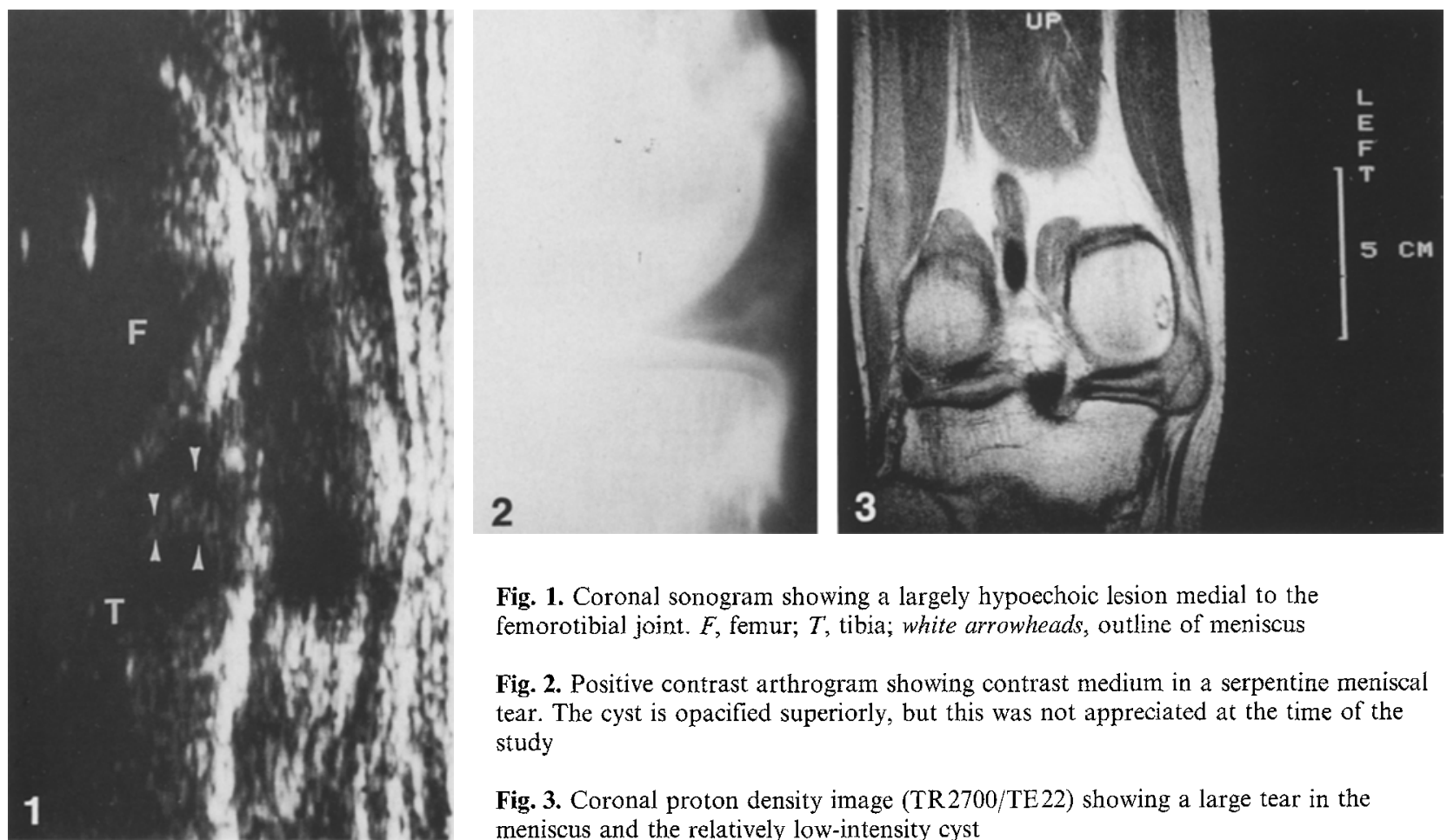

Fig. 1. Coronal sonogram showing a largely hypoechoic lesion medial to the femorotibial joint. $F$, femur; $T$, tibia; white arrowheads, outline of meniscus

Fig. 2. Positive contrast arthrogram showing contrast medium in a serpentine meniscal tear. The cyst is opacified superiorly, but this was not appreciated at the time of the study

Fig. 3. Coronal proton density image (TR2700/TE22) showing a large tear in the meniscus and the relatively low-intensity cyst
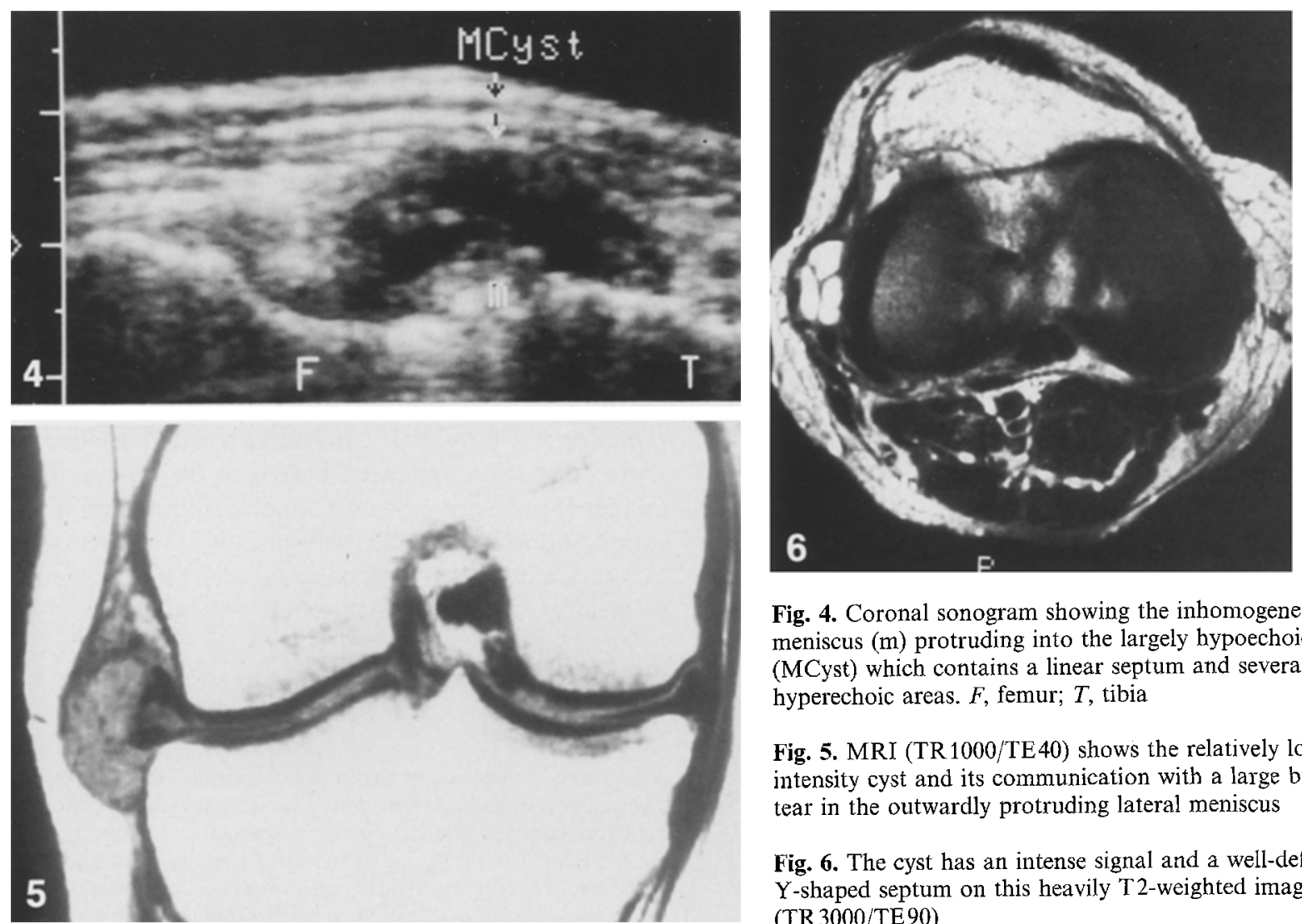

Fig. 4. Coronal sonogram showing the inhomogeneous meniscus $(\mathrm{m})$ protruding into the largely hypoechoic lesion (MCyst) which contains a linear septum and several small hyperechoic areas. $F$, femur; $T$, tibia

Fig. 5. MRI (TR1000/TE40) shows the relatively low intensity cyst and its communication with a large bulbous tear in the outwardly protruding lateral meniscus

Fig. 6. The cyst has an intense signal and a well-defined $\mathrm{Y}$-shaped septum on this heavily T2-weighted image (TR3000/TE90) 

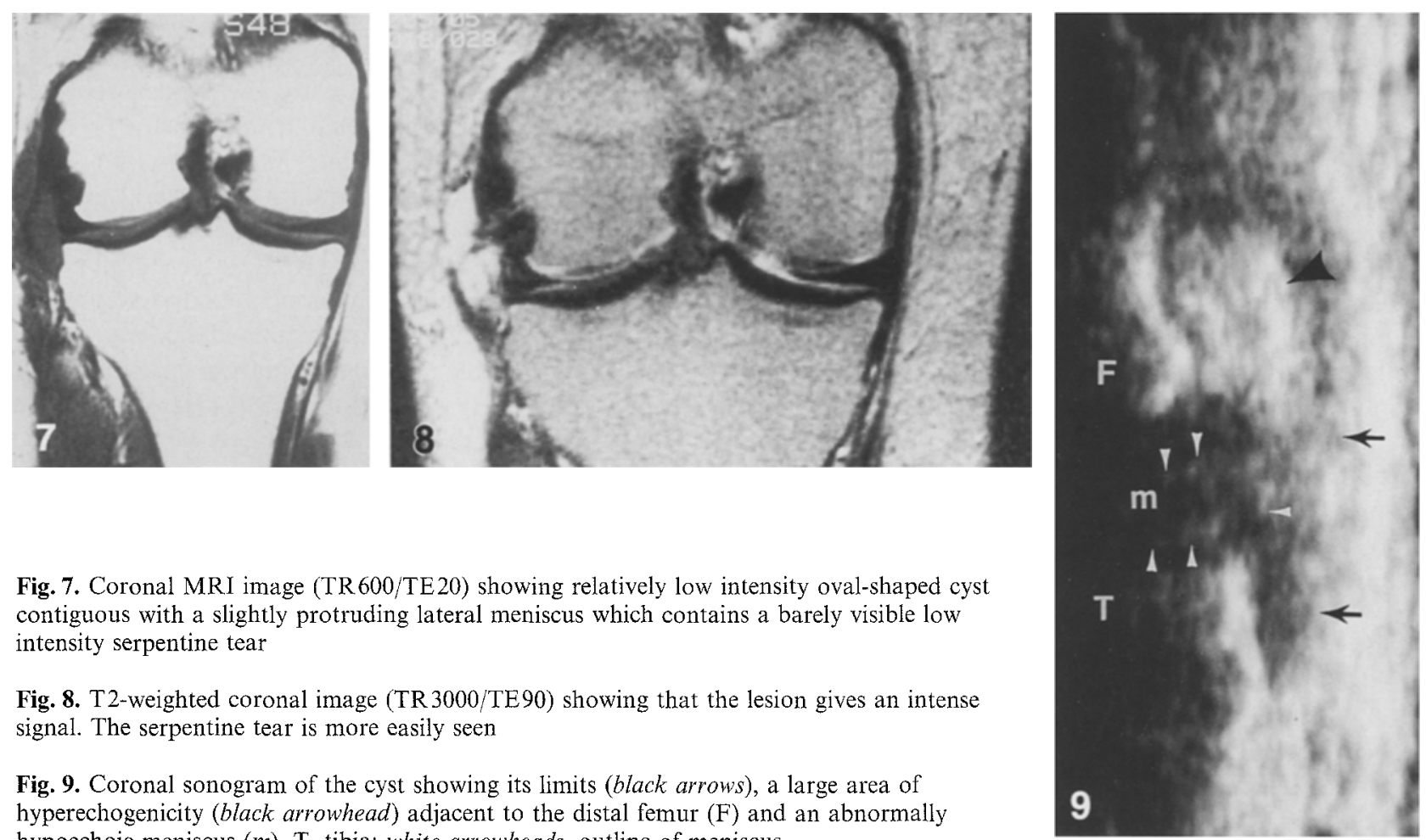

Fig. 7. Coronal MRI image (TR600/TE20) showing relatively low intensity oval-shaped cyst contiguous with a slightly protruding lateral meniscus which contains a barely visible low intensity serpentine tear

Fig. 8. T2-weighted coronal image (TR 3000/TE90) showing that the lesion gives an intense signal. The serpentine tear is more easily seen

Fig. 9. Coronal sonogram of the cyst showing its limits (black arrows), a large area of hyperechogenicity (black arrowhead) adjacent to the distal femur $(\mathrm{F})$ and an abnormally hypoechoic meniscus $(m)$. T, tibia; white arrowheads, outline of meniscus

cated with a collection of contrast medium medial to the joint (Fig. 2). A proton density sequence (TR 2700/TE22) obtained on a Siemens $1.5 \mathrm{~T}$ magnet showed the lesion to have moderate intensity, higher than that of muscle and less than that of fat, and to contain a septum. The lesion was contiguous with the meniscus, which contained a horizontal tear (Fig. 3). On T2-weighted scans (not shown) the cyst had very high relative intensity. The cyst and tear were confirmed at surgery.

\section{Case 2}

A 56-year-old woman had lateral right knee pain and swelling for 3 months without a history of trauma. Ultrasound showed a crescentshaped hypoechoic area containing a septum and focal areas of hyperechoic matter (Fig. 4). MRI using a 0.35 T magnet (Diasonics) showed a lesion of relatively low intensity contiguous with a horizontal area of similar intensity in the meniscus on TR 1000/TE40 sequences (Fig.5). A T2-weighted axial image (TR2000/TE 80) showed a $Y$-shaped septum dividing a relatively high-intensity lesion (Fig. 6). The meniscus clearly protruded into the cyst on both ultrasound and coronal MRI. As symptoms have remained mild, the patient has not sought surgery.

\section{Case 3}

A 31-year-old female factory worker had 6 months of intermittent swelling, made worse by exertion, on the lateral aspect of the right knee. The swelling was first noticed after a direct blow to the lateral margin of the joint. Examination showed a diffuse, soft swelling on the lateral aspect of the femorotibial joint margin. The referring physician was unaware of the diagnosis and had suspected that a neoplasm was present. MRI with a $1.5 \mathrm{~T}$ magnet (General Electric Signa) showed an oval collection which was of relatively low intensity on T1-weighted images (Fig. 7) and high intensity on T2-weighted images (Fig. 8). The lesion was adjacent to the meniscus, which contained a serpentine tear that had a relatively high-intensity signal on T2-weighted images. U1trasonography showed an abnormally hypoechoic meniscus protruding into the lesion, which contained a large focus of high echogenicity (Fig. 9). A subtotal meniscectomy was performed and confirmed the meniscal tear and cystic degeneration.

\section{Discussion}

There has not been an exact definition in the literature of what constitutes a meniscal cyst. However, recent studies have shown that a meniscal tear is present in every case of cyst $[1,5,8,14]$ and other authors had previously noted a frequent association between incomplete transverse or oblique tears and cysts, particularly of the lateral meniscus [17]. Therefore, a meniscal cyst could be defined as an encapsulated mass containing synovial-like fluid [1] continuous with the meniscus from which it arises and a horizontally oriented tear within the semilunar fibrocartilage.

Using this definition, fluid-filled cysts not attached to the meniscus are separate entities. These are often ganglion cysts which are the result of degeneration within a tendon sheath, a ligament, or joint capsule and are not associated with tears of the menisci.

Smillie pointed out that cysts of the medial men- 
iscus are more often present within the substance of the fibrocartilage than are lateral cysts because of the firmer attachment of the medial fibrocartilage to the capsule, an observation confirmed by Ferrer-Roca and Vilalta [8]. For the same reason, medial cysts may occasionally lie at some distance from the meniscus because the close anatomic relationship between the meniscus and the collateral ligament does not allow for much expansion between them. If a large cyst should lie posteriorly it will be displaced progressively by the collateral ligament during knee flexion towards the popliteal fossa where it will be connected to the meniscus by a long pedicle and may be mistaken for a popliteal cyst or ganglion cyst [17]. Cysts of the lateral meniscus tend to remain close to the periphery of the fibrocartilage because of the potential space due to the popliteus tendon and are therefore more likely to present with a palpable mass to the side of the joint.

Recently it has been suggested that cysts of the meniscus are formed by fluid that has been forced along a horizontal tear and accumulates in the meniscocapsular region $[1,8]$. The cysts are a product of meniscal damage due, in the majority of young patients, to trauma. Similarly, these cysts have been noted in patients with discoid menisci $[2,15]$ because the abnormally thick fibrocartilage is prone to injury. As many as half the patients have a history of trauma $[1,2]$. Sometimes the cyst is found after a direct injury as in a case of a miner who received a blow to the side of the knee where a meniscal cyst subsequently developed [15]. Our third case reported such an injury.

In Smillie's large series, meniscal cysts were 3.3 times more common in lateral than medial menisci, but as there were many more resected medial menisci, the incidence of cysts in surgically removed specimens of the lateral meniscus is nine times greater. The commonest site is the periphery of the middle third of the lateral meniscus, and the cysts are usually multiple [17]. The male to female preponderance seen in most series $[14,17]$ is probably due to the more frequently damaged menisci in males and the greater number of arthrograms and meniscectomies performed in men. The mean age of affected individuals is 36 years, the same as for patients having knee arthrograms [14], and, although most patients are in the third and fourth decades, the age range of reported cases varies from 5 years in the case of a girl with a discoid meniscus [2] to 72 [14]. They usually present with a palpable bulge to the side of the femorotibial joint and nearly all patients will admit to associated knee pain [2]. The pain, which may be the only complaint, is typically dull and is made worse by exercise $[9,18]$. Locking and giving way are unusual. The characteristic feature of the swelling is that it varies in size depending on the position of the knee, being largest in extension and smallest in flexion [16].

The size of the cysts varies, the largest reported being $7 \times 6 \times 4 \mathrm{~cm}$ in the medial meniscus [11]. Most cases do not produce an abnormality on plain radiographs, although Golding points out several features that may be present in cases of cyst of the lateral meniscus. These are widening to the lateral joint space, which may have been due to associated discoid menisci unsuspected by him; a faint rounded opacity, sometimes with a visible medial cyst margin; erosion of the bone immediately below the lateral articular margin due to extrinsic pressure by the cyst (a finding also reported by Enis and Ghandur-Mnaymneh [7]); and a deposit of calcification in the cyst. Underexposed films $[10,11]$ and intercondylar views may be necessary to demonstrate these abnormalities [10].

Arthrography has been the usual way in which cysts of the menisci have been demonstrated preoperatively [14]. However, the characteristic appearance of a serpentine tear in the meniscus, with a club-shaped pooling of contrast medium peripherally, may not be appreciated and the diagnosis may be missed. The cyst may only show filling with air on films made many hours later (filling occurred at 72 hours in one case) [4].

Computed tomography has also been used to confirm the presence of meniscal cysts [6] and is able to show most meniscal tears if $2 \mathrm{~mm}$ contiguous sections are made through the menisci [12]. MRI is able to show the meniscal cysts on coronal, sagittal, and axial scans. However, coronal scans were best in our cases for showing the cysts and the communicating horizontal tear. These tears were easily seen on both T1- and T 2weighted images in the cases described in this report. The cysts were all highly intense on $\mathrm{T} 2$-weighted scans.

The normal appearances of menisci on real-time sonograms have been reviewed [13]. The normal meniscus is homogeneously echogenic and triangular with the apex pointing inwardly. Ultrasound in our cases showed a predominantly hypoechoic lesion adjacent to a meniscus. In the two cases of lateral meniscal cyst, sonography showed an abnormal meniscus protruding into the cyst. In these cases also, there were foci of highly echogenic material within the cyst, and a septum was seen in one.

The cases described in this report show MRI to be a good means of demonstrating cysts and the accompanying tears in the menisci. However, we also show that ultrasound can demonstrate the cyst and debris within it, and is also able to show the abnormal meniscus in cases of lateral meniscal cyst.

We predict that the use of high-resolution ultrasound, computed tomography, and MRI in the investigation of suspected tumors of the lower limbs will 
lead to the diagnosis of an increasing number of meniscal cysts in the future. MRI has thus far proven to be accurate in the diagnosis of meniscal cysts and other lesions of the knee. It is, however, a time consuming, expensive test. Ultrasound may prove to be an alternative means of diagnosing meniscal cysts. It is able to show the relationship of the cyst to the meniscus and sometimes the tear. Studies with larger numbers of patients are needed to determine the accuracy of ultrasound in diagnosing meniscal cysts and differentiating them from other cystic pathology in the knee. However, in places where access to MRI is restricted or nonexistent, ultrasound may be helpful in establishing a diagnosis of this condition.

\section{References}

1. Barrie HJ (1979) The pathogenesis and significance of meniscal cysts. J Bone Joint Surg [Br] $61: 184$

2. Becton L, Young HH (1965) Cysts of semilunar cartilage of the knee. Arch Surg 90:708

3. Breck LW (1954) Cysts of the semilunar cartilages of the knee. Clin Orthop 3:29

4. Buckwalter JA, Dryer RF, Mickelson MR (1979) Arthrography in juxta-articular cysts of the knee. J Bone Joint Surg [Am] $61: 465$

5. Burk DL, Dalinka MK, Kanal E, Schiebler ML, Cohen EK,
Provok RJ, Gefter WB, Kressel HY (1988) Meniscal and ganglion cysts of the knee: MR evaluation. AJR 150:331

6. Chen W-C, Wu J-J, Chang C-Y, Chen B-F, Vang D-J (1987) Computed tomography of a meniscal cyst. Orthopedics 10:1569

7. Enis JE, Ghandur-Mnaymeneh L (1979) Cyst of the lateral meniscus causing erosion of the tibial plateau. J Bone Joint Surg [Am] 61:442

8. Ferrer-Roca O, Vilalta C (1980). Lesions of the meniscus. Part II: horizontal cleavages and lateral cysts. Clin Orthop 146: 301

9. Gallo GA, Bryan RS (1968). Cysts of the semilunar cartilage of the knee. A report of sixteen cases including arthrographic study. Am J Surg 116:65

10. Golding C (1960) Museum pages II. Cysts of the lateral semilunar cartilage of the knee. J Bone Joint Surg [Br] 42: 144

11. McGehee FO, Cameron BM (1955) Large cyst of the medial meniscus of the knee. Report of a case. J Bone Joint Surg [Am] 37:1281

12. Passariello R, Trecco F, de Paulis F, Masciocchi C, Bonanni G, Zobel BB (1985) Meniscal lesions of the knee joint: CT diagnosis. Radiology 157:29

13. Richardson ML, Selby B, Montana MA, Mack LA (1988) Ultrasonography of the knee. Radiol Clin North Am 26:63

14. Schuldt DR, Wolfe RD (1980) Clinical and arthrographic findings in meniscal cysts. Radiology 134:49

15. Smillie IS (1951) Injuries of the knee joint. 2nd edn. Churchill Livingstone, Edinburgh, $\mathrm{p} 48$

16. Smillie IS (1951) Injuries of the knee joint. 2nd edn. Churchill Livingstone, Edinburgh, p 89

17. Smillie IS (1970) Injuries of the knee joint. 4th edn. Williams and Wilkins, Baltimore, $\mathrm{p} 48$

18. Smillie IS (1970) Injuries of the knee joint. 4th edn. Williams and Wilkins, Baltimore, $p 85$ 